Article

\title{
High-fructose diet initially promotes increased aortic wall thickness, liver steatosis, and cardiac histopathology deterioration, but does not increase body fat index
}

\author{
Dian Handayani, ${ }^{1}$ Erlinda Febrianingsih, ${ }^{1}$ Adelya Desi Kurniawati, ${ }^{1}$ Inggita Kusumastuty, ${ }^{1}$ \\ Shafira Nurmalitasari, ${ }^{1}$ Rahma Micho Widyanto, ${ }^{1}$ Diah Novida Oktaviani, ${ }^{1}$ \\ Alma Maghfirotun Innayah, ${ }^{2}$ Etik Sulistyowati $^{3}$ \\ ${ }^{1}$ Department of Nutrition, Faculty of Medicine, Universitas Brawijaya, Malang; ${ }^{2}$ Master Program in Biomedical \\ Sciences, Faculty of Medicine, Universitas Brawijaya, Malang; ${ }^{3}$ Nutrition Department, Polytechnic of Health, \\ Malang, Indonesia
}

\begin{abstract}
Background: Dietary fats and fructose have been responsible for inducing obesity and body tissues damage due to the consequence of metabolic syndrome through several mechanisms. The body fat index (BFI) is one of the anthropometric measures used to detect obesity in rats. This study aims to examine the correlation between high-fat high-fructose diet and liver steatosis cell count, early atherosclerosis characteristics, and body fat index (BFI) in Sprague Dawley rats.

Design and methods: This was an experimental design using 2 groups of 12-weeks-old Sprague Dawley (SD) rats. The control group received a standard diet and tap water beverages for 17 weeks. The intervention group was fed with high-fat diet from modified AIN 93-M and additional 30\% fructose drink. We analyzed the foam cell count, aortic wall thickness, cardiac histopathology, and liver steatosis cell count after the sacrifice process.

Results: The rats in the intervention group had a higher aortic wall thickness, liver steatosis, and foam cell count $(+125 \%$, $\mathrm{p}<0.01 ;+317 \%, \mathrm{p}<0.01$ and $+165 \%, \mathrm{p}<0.01$ respectively) compared to the control group. The intervention group also showed higher mononuclear inflammatory and hypertrophic cell count. A significant positive correlation was found between dietary fructose with premature atherosclerosis by increasing foam cell count $(\mathrm{r}=0.66)$ and aortic wall thickness $(\mathrm{r}=0.68)$. In addition, $30 \%$ dietary fructose increased liver steatosis $(r=0.69)$ and mononuclear inflammatory cardiac cell count $(r=0.61)$. Interestingly, the intervention had no effect on the body fat index ( $\mathrm{p}>0.5 ; \mathrm{r}=0.13)$.

Conclusions: Dietary fat and fructose consumption for 17 weeks promotes atherosclerosis, liver steatosis, and cardiac histopathology alteration without increasing BFI.
\end{abstract}

\section{Introduction}

Obesity is the most important component of metabolic syndrome, as a result of imbalance between energy intake and expenditure, followed by an increase in adiposity. This condition is characterized by the accumulation of visceral fat followed by certain conditions, such as insulin resistance, lipid profile alteration, and the elevation of chronic inflammation marker. Overweight and obesity are among the risk factors in liver steatosis and atherosclerosis, as well as structural and functional changes of the heart, which cause heart failure. ${ }^{1-3}$ Atherosclerosis is known as chronic inflammation disease leading to tunica intima thickening and arterial rigidness, which starts with foam cell formation, atheroma, leucocyte accumulation, smooth muscle proliferation, and, finally, atherosclerosis formation. ${ }^{4}$ Liver steatosis, specifically non alcoholic fatty liver disease, is a disease characterized by triglyceride accumulation in the liver. It encompasses a variety of histopathological findings, from non-alcoholic steatohepatitis to fibrosis and cirrhosis hepatic, which may develop into hepatocellular carcinoma. ${ }^{5}$ Meanwhile, obesity leads to heart failure through several mechanisms, which includes hemodynamic changes and activation of the renin-angiotensin-aldosterone (RAAS) system. In addition, heart failure can be characterized by cardiac histopathology alteration, which is promoted by platelet aggregation, endothelial dysfunction, and interstitial cardiac. ${ }^{3}$

Overweight and obesity among Indonesian adults have gradually increased from $8.6 \%$ in 2007 to $13.6 \%$ in 2018 for overweight and $10.5 \%$ in 2007 to $21.8 \%$ in 2018 for obesity. Central obesity has also increased in adults, from $18.8 \%$ in 2007 to $31 \%$ in 2018.6 The WHO showed that overweight prevalence in adults reached $39 \%$ and $13 \%$ of adults were obese in 2016. Furthermore, 41 million children under the age of 5 were obese or overweight and over 340 million children and adolescents were overweight or obese in

Significance for public health

Obesity is a major health problem that is a powerful inducer of non-communicable disease (NCD) both in the national and global level of the human population age groups. Based on this study, consumption of fructose also poses an increased risk in developing metabolic syndrome, which differs from the current understanding of dominantly caused by excessive intake of fat. Furthermore, high fructose consumption promotes early signs of cardiovascular and liver disease without significant manifestation in anthropometry characteristics. It is believed that these results are important and will give readers a broader view on combating obesity epidemics from a dietary and nutritional perspective, which may also contain additional reference for nutrition education or dietary advice for the prevention of obesity. 
2016. ${ }^{7}$ Obesity as well as other metabolic conditions such as high blood pressure and high blood glucose were the key risk factors of non-communicable disease (NCD). It is estimated that NCDs account for $71 \%$ of the 57 million global deaths in productive age (30-70 years old), which is consisted of $31 \%$ death caused by cardiovascular disease and 3\% death caused by diabetes. ${ }^{8}$

Several studies regarding obesity and excessive energy intake were conducted. ${ }^{9-11}$ These conditions are linked to increased consumption of high fat in fast-food and high fructose in soft drink. Almost all types of soft-drink have some sorts of glucose or fructose content. Fructose is commonly used as a sweetener in these beverages due to its sweet flavor, low cost, and ability to promote a long shelf-life. Recent studies have also identified fructose as the cause of obesity and other metabolic diseases. ${ }^{12,13}$ High fructose intake is also linked to the increase of superoxide production, which leads to endothelial damage. ${ }^{14}$

In connection with the recent trend of increased consumption of soft-drinks and the used of fructose, there is a risk of excessive consumption of fructose in relation to obesity, atherosclerosis, liver steatosis, and the change in cardiac histopathology need further investigation. This study examined the correlation between high fructose diet and early atherosclerosis, which is characterized by increased foam cell count, aortic wall thickness, liver steatosis cell count, cardiac histopathology, and body fat index (BFI). More specifically, this study systematically examined if high fat-high fructose diet (HFFD) induced obesity first or body tissue damage as the consequence of metabolic syndrome. Obesity was determined using anthropometric measurement, while body tissues damage was determined by examine aortic wall thickness; foam cell count; liver steatosis cell count; mononuclear inflammatory cardiac cell count; and hypertrophic cardiac cell count.

\section{Design and Methods}

This research was experimentally designed, and all procedures were approved by the research ethics committee of Faculty of Medicine, Universitas Brawijaya, Indonesia (368/EC/KEPK/10/2017). Thirty-six (36) male Sprague Dawley (SD) obesity model rats aged 8 weeks were given four weeks acclimatization period, which included one-rat-one-cage housing and normal diet. The 36 rats were divided randomly into two groups $(\mathrm{n}=18) .{ }^{15}$ The control group was fed normal or standard diet containing $31 \%$ protein; $26 \%$ fat; and $43 \%$ carbohydrate. The other group was fed with HFFD containing 52\% fat; $22 \%$ protein; and $29 \%$ carbohydrate pellets with an addition of $30 \%$ fructose solution. ${ }^{16,17}$ The energy density was $4.21 \mathrm{kcal}$ and $5.08 \mathrm{kcal}$ for control group and HFFD group respectively. The dietary intervention was carried out for 17 weeks (September 2017-January 2018). The food, which was modified from AIN-93 M, was produced from corn starch, dextrinized cornstarch, sucrose, soybean oil, casein, egg-white flour, gelatin, mineral and vitamin mix AIN, L-cysteine, and choline bitartrate. The composition of the two diets is shown in Table 1 .

\section{Food intake, body weight gain, and body composition}

The animals are weighed weekly during the 17 weeks intervention period. Food consumption was calculated by weighing the amount of total food $(\mathrm{g})$ given to the rats and subtracting the remaining food $(\mathrm{g})$ in the cage every $24 \mathrm{~h}$. After the intervention period, rats were sacrificed by ketamine injection. We dissected out and weighed the white adipose tissue (WAT), which is comprised of visceral fat (epididymal, perirenal, and omental fat) and subcutaneous fat (inguinal fat). The BFI was determined as the total amount of visceral and subcutaneous fat deposits per $100 \mathrm{~g}$ body weight. ${ }^{18}$

\section{Liver steatosis, aortic wall thickness, foam cell count, and cardiac histopathology}

Liver steatosis cell count was examined using Hematoxylin \& Eosin (HE) staining. Briefly, using a cryostat, the frozen rat livers were placed in a $10 \mu \mathrm{m}$-portion cut and fixated in ice-cold $10 \%$ formalin. The livers were air dried for $60 \mathrm{~min}$ and rinsed 3 times in distilled water. The slices were differentiated in $85 \%$ propylene glycol solution for $5 \mathrm{~min}$ and stained in HE for $30 \mathrm{~s}$. The specimens were washed in running tap water for $3 \mathrm{~min}$, mounted onto slides using glycerin jelly, and covered.

Aortic wall samples were taken, rinsed in aquades, and fixed in $10 \%$ formalin for at least 7 hours. The aortic wall was sliced in 2$3 \mathrm{~mm}$ sections and treating using Tissue Tex Processor for $90 \mathrm{~min}$. These tissues were treated for paraffin embedding, then sectioned using microtome with 3-5 $\mu$ thin section and went in for deparaffinization process. The specimens were stained with standard HE staining, and the wall thickness of the aorta was measured from the tunica intima (TI) op to tunica media (TM) in 8 fields of view in a clockwise direction (at $12.00 ; 13.30 ; 15.00 ; 16.30 ; 18.00 ; 19.30$; $21.00 ; 22.30$ ). For the foam cell count, the specimens were examined from all fields of view at the cross-section of the aortic wall. The foam cells counted were those located in the tunica intima and tunica media. The histological examinations were carried out at a magnification of $\times 400$ using a light microscope. In terms of cardiac histopathology analysis, the variables examined were mononuclear inflammatory heart count, hypertrophic heart count, and fibrous formation. The major steps of histopathology sample preparation were similar and included the process of paraffin embedding followed by a deparaffinization process. The samples for mononuclear inflammatory cell and hypertrophic cell examination were stained with standard HE staining and examined under $\times 400$ and $\times 1000$ magnification, while the fibrotic formation examination samples were stained using Masson Trichome staining and examined under $\times 200$ magnification.

\section{Statistical analysis}

Food intake, body weight gain, BFI, liver steatosis cell count, aortic wall thickness, foam cell count, mononuclear inflammatory cardiac cell count, and hypertrophic cardiac cell count were presented as mean and standard errors. BFI, total carbohydrate, and protein intake data were transformed to $\log 10$ values and liver steatosis, foam cell count data were converted to square root values to achieve normality before further testing. Total carbohydrate intake, protein intake, liver steatosis cell count, foam cell count, and cardiac histology were analyzed using Mann-Whitney U test, while the other comparison tests were analyzed using the independent t-test. The differences are considered significant when $\mathrm{p}<$

Table 1. Composition of diet.

\begin{tabular}{lcc} 
Component & \multicolumn{3}{c}{ Group } \\
& Control group & HFF group \\
Carbohydrate (\%) & 42.87 & 29.39 \\
Protein (\%) & 31.32 & 21.81 \\
\hline Fat (\%) & 25.81 & 51.64 \\
Fructose (\%) & - & 30 \\
\hline Energy density $(\mathrm{kcal} / \mathrm{g})$ & 4.21 & 5.08 \\
\hline
\end{tabular}

HFFD, high-fat fructose diet (modified from ${ }^{16,17}$ ). 
0.05. Simple associations between energy and fat intake with dependent variables were calculated using Pearson's correlation coefficient, while the association between total carbohydrate and protein intake data and the dependent variables were calculated using Spearman's correlation coefficient. All of the statistical tests were performed using SPSS Software (ver. 16.0, SPSS Inc., Chicago, IL, USA).

\section{Results and Discussions}

The final examination was carried out on 14 rats in the control group and 16 rats in HFFD group from 36 rats in the initial study caused by death during the intervention. The amount of food energy intake was significantly higher in HFFD group $(+134 \%$, $\mathrm{p}=0.000)$. Rats on HFFD group had $+127 \%$ higher fat intake and $56 \%$ lower protein intake compared to rats in control group $(\mathrm{p}=0.001$ and $\mathrm{p}=0.000$ for fat and protein intake respectively). Due to the additional fructose solution in the HFFD group, the carbohydrate intake in the HFFD group was significantly higher $(+208 \%$, $\mathrm{p}=0.000$ ) than in the control group. We found no significant difference in the body weight gain between the two diet groups (Table 2 ). No difference in the body fat index was reported between control group and HFFD group.

The number of liver steatosis cells was significantly higher in the HFFD group $(+317 \%$; $=0.000)$ than in the control group. A significantly greater aortic wall thickness and a significantly higher foam cell count in HFFD compared to control group was also reported (Figure 1; $+125 \%$; $\mathrm{p}=0.000$ and $+165 \%$; $\mathrm{p}=0.000$ for aortic wall thickness and foam cel,1 respectively). The HFFD group showed a higher mononuclear inflammatory cardiac cell count $(+120 \%, p=0.000)$ and hypertrophic cardiac cell count $(+118 \%$, $\mathrm{p}=0.001)$. The presence of fibrotic cell was also observed in cardiac histopathology examination of HFFD group (Figure 1).

The total energy intake was reported to be positively correlated (Figure 2) with body fat index $(\mathrm{R}=0.366 ; \mathrm{p}=0.047)$, body weight gain $(\mathrm{R}=0.496 ; \mathrm{p}=0.005)$, aortic wall thickness $(\mathrm{R}=0.708$; $\mathrm{p}=0.000)$, foam cell count $(\mathrm{R}=0.612 ; \mathrm{p}=0.000)$, liver steatosis cell count $(\mathrm{R}=0.650 ; \mathrm{p}=0.000)$, and mononuclear inflammatory cardiac

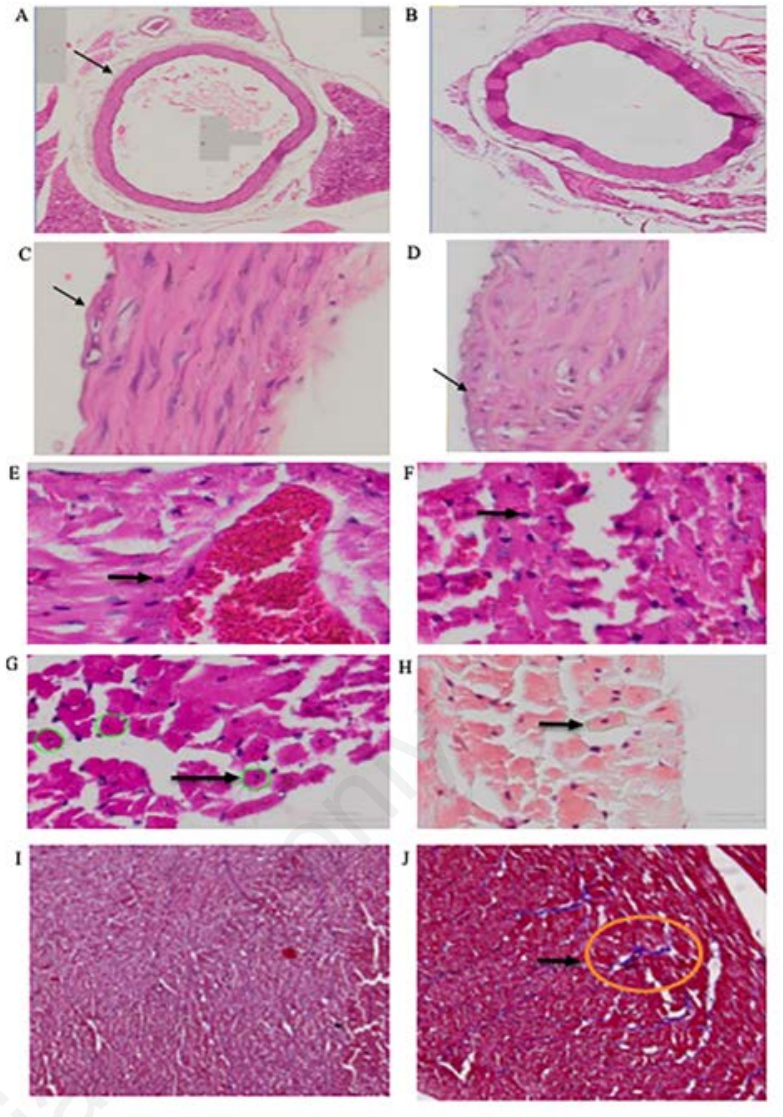

Figure 1. The effect of HFFD on thickness of aorta tissues and foam cell count of Sprague Dawley Rats. A) Aorta tissues of control diet. B) Aorta tissues of HFFD. C) Foam cell of control diet. D) Foam cell of HFFD. E) Mononuclear inflammatory cardiac cell of control diet. F) Mononuclear inflammatory cardiac cell of HFFD. G) Hypertrophic cardiac cell of control diet. H) Hypertrophic cardiac cell of HFFD. I) Absence of fibrotic formation in cardiac cell of control diet group. J) Fibrotic formation in cardiac cell of HFFD group.

Table 2. Food intake, body weight gain, body fat index, liver steatosis, and cardiac histopathology of rats.

\begin{tabular}{|c|c|c|c|}
\hline Variables & Control $(n=14)$ & HFFD $(n=16)$ & Independent $t$-test \\
\hline Energy intake (kcal/day) & $50.6 \pm 6.8$ & $67.7 \pm 6.4$ & 0.000 \\
\hline Fat intake (g/day) & $1.4 \pm 0.2$ & $1.8 \pm 0.3$ & 0.001 \\
\hline Protein intake (g/day) & $3.9 \pm 0.5$ & $1.7 \pm 0.3$ & 0.000 \\
\hline Total carbohydrate intake (g/day) & $5.4 \pm 0.7$ & $11.3 \pm 1.1$ & 0.000 \\
\hline Body weight gain (g) & $15.8 \pm 23.7$ & $31.3 \pm 26.3$ & NS \\
\hline Body fat index & $27.4 \pm 10.2$ & $29.3 \pm 12.9$ & NS \\
\hline Liver steatosis cell count & $19.6 \pm 20.0$ & $62.2 \pm 23.3$ & 0.000 \\
\hline Aortic wall thickness & $103.6 \pm 14.1$ & $129.9 \pm 6.6$ & 0.000 \\
\hline Foam cell count & $3.3 \pm 1.0$ & $5.4 \pm 1.8$ & 0.000 \\
\hline Mononuclear inflammatory cardiac cell count & $40.4 \pm 4.1$ & $48.8 \pm 5.0$ & 0.000 \\
\hline Hypertrophic cardiac cell count & $1527.3 \pm 220.8$ & $1812.8 \pm 202.6$ & 0.001 \\
\hline Total cholesterol & $51 \pm 11.5$ & $61.8 \pm 15.0$ & 0.038 \\
\hline Plasma Triglyceride & $36.5 \pm 11.2$ & $40.2 \pm 12.4$ & NS \\
\hline Plasma HDL & $18.9 \pm 4.7$ & $23.3 \pm 7.8$ & NS \\
\hline Plasma LDL & $24.7 \pm 8.0$ & $30.2 \pm 8.3$ & NS \\
\hline
\end{tabular}

Values are means \pm SEM, $n=30$; HFFD, high-fat fructose diet; HDL, high density lipoprotein; LDL, low density lipoprotein; NS, not significant. 
cell count $(\mathrm{R}=0.529 ; \mathrm{p}=0.003)$. There was a positive association (Figure 3) between fat intake and body fat index $(\mathrm{R}=0.576$; $\mathrm{p}=0.001)$, body weight gain $(\mathrm{R}=0.652 ; \mathrm{p}=0.000)$, aortic wall thickness $(R=0.550 ; p=0.002)$, foam cell count $(R=0.521 ; p=0.003)$, and liver steatosis cell count $(\mathrm{R}=0.579 ; \mathrm{p}=0.001)$. The total carbohydrate intake including carbohydrate from food and fructose from drink also showed a positive association (Figure 4) with body weight gain $(\mathrm{R}=0.410 ; \mathrm{p}=0.024)$, aortic wall thickness $(\mathrm{R}=0.767$; $\mathrm{p}=0.000)$, foam cell count $(\mathrm{R}=0.657 ; \mathrm{p}=0.000)$, liver steatosis cell count $(\mathrm{R}=0.657 ; \mathrm{p}=0.000)$, and mononuclear inflammatory cardiac cell count $(\mathrm{R}=0.613 ; \mathrm{p}=0.000)$, although there was no statistical association between total carbohydrate intake and body fat index and hypertrophic cardiac cell count.

The protein intake showed no statistical association with body fat index and body weight gain, although it was negatively associated with aortic wall thickness $(\mathrm{R}=-0.622 ; \mathrm{p}=0.000)$, foam cell count $(\mathrm{R}=-0.503 ; \mathrm{p}=0.005)$, liver steatosis cell count $(\mathrm{R}=-0.628$; $\mathrm{p}=0.000)$, mononuclear inflammatory cardiac cell count $(\mathrm{R}=$ $-0.586 ; \mathrm{p}=0.001)$, and hypertrophic cardiac cell count $(\mathrm{R}=-0.512$; $\mathrm{p}=0.004)$. This study showed no difference in body weight gain (BWG) and BFI between the two groups. This is in line with the studies conducted by Ble-Castillo et al., and Pang et al., which reported that BWG is was not significantly higher in rats administered with high-fat and high-fructose diet than in the control group. ${ }^{19,20}$ Despite the lack of significant differences in BWG and BFI after HFFD treatment, this study showed that energy and fat intake were associated with increase in BWG and BFI while fructose intake was only positively correlated with BWG. This is similar to the results of previous studies that showed that higher levels of energy and fat in HFFD promoted obesity, which is characterized by higher fat mass, visceral fat, and body fat percentage. ${ }^{13,21,22}$ Several previous studies also claim that higher energy, fat, and
A

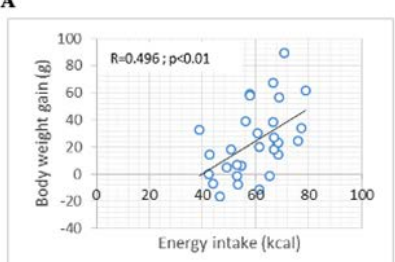

C

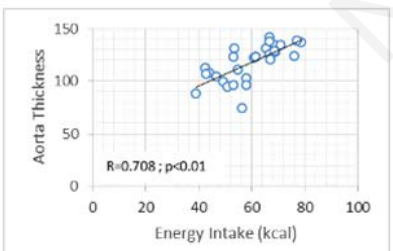

E

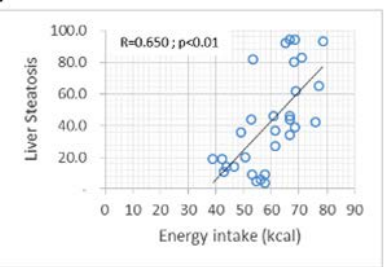

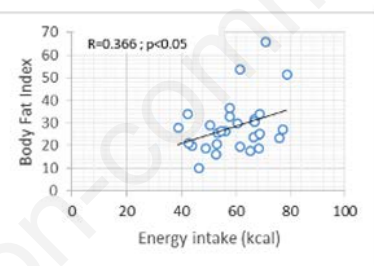

D

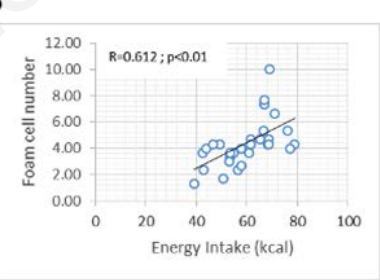

$\mathbf{F}$

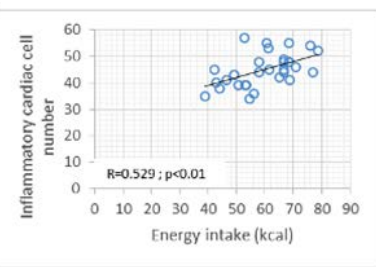

Figure 2. Significant correlations were found between energy intake and (A) body weight gain; B) body fat index; C) aorta thickness; D) foam cell number; E) liver steatosis; F) mononuclear inflammatory cardiac cell count.

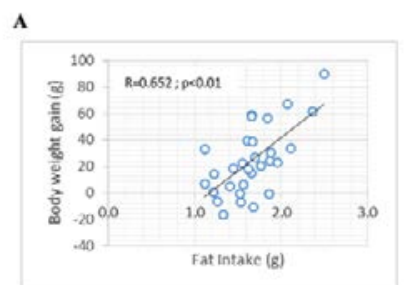

B
C

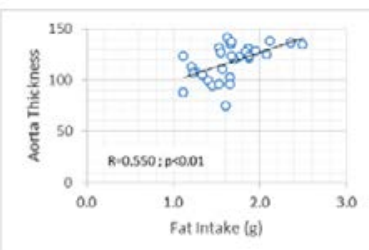

E

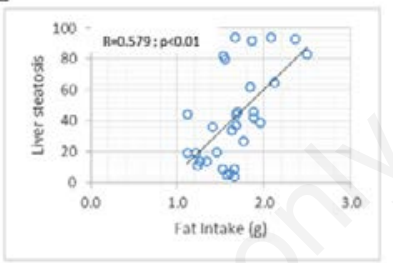

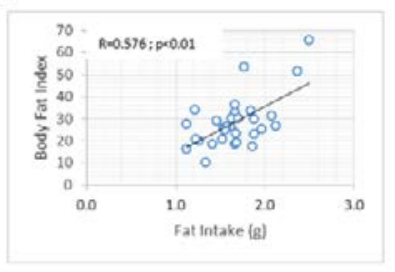

D

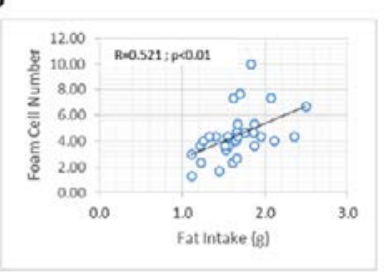

Figure 3. Significant correlations were found between fat intake and (A) body weight gain; B) body fat index; C) aorta thickness; D) foam cell number; E) liver steatosis.

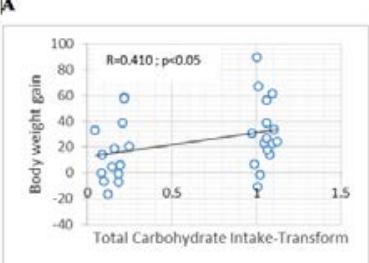

C

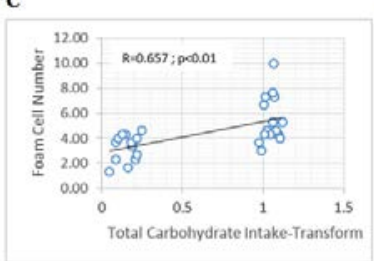

E

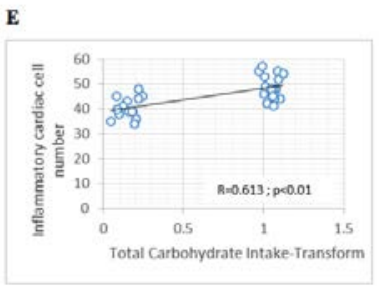

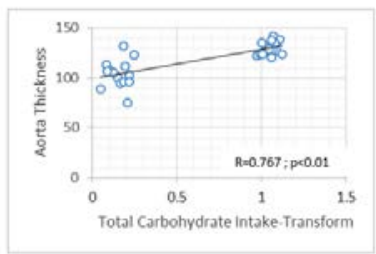

D

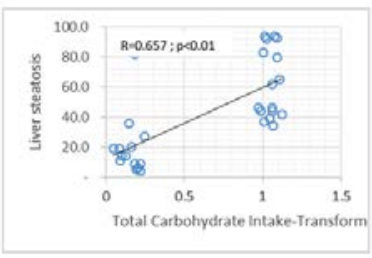

Figure 4. Significant correlations were found between carbohydrate intake and (A) body weight gain; (B) aorta thickness; (C) foam cell number; (D) liver steatosis; (E) mononuclear inflammatory cardiac cell number. 
fructose intake not only promote 1.3-1.4× higher BWG increase than the normal group but also increased body fat. ${ }^{13,20,21}$

Several factors that can explain these different results are diet composition and duration of intervention. In this study, HFFD containing 51\% fat and 30\% fructose drink administered during a 17 week period produced no different effect on BFI and BWG. Similar result is also demonstrated by another study using $60 \%$ fat and $20 \%$ of glucose addition for 12 weeks. Meanwhile, other studies using a variety of fat and fructose composition such as $45 \%$ fat for 35 weeks, $15 \%$ fructose for 24 weeks, and high-fat and sucrose intervention for 60 weeks promoted BWG and body fat increase. ${ }^{13,21,22}$ Some high-fat diet ( $45 \%$ fat or $60 \%$ fat) increased $20-30 \%$ body weight after a feeding period of $16-20$ weeks, while the manifested picture of obesity such as increased fat mass started to develop after 16 weeks. ${ }^{23}$ However, fructose is more likely to cause body weight gains if consumed over a long period of time. Furthermore, it was known that HFFD had no adverse effects on glucose and fat metabolism compared to a high-fat diet alone, while high-saturated fat diet might have more significant effect on body weight gain although having similar calorie content. ${ }^{20}$ Therefore, the duration of intervention, macronutrient ratio, and fructose drink ratio are also important both for body weight and body fat gain.

The histological examination of the liver showed that the HFFD group had a significantly higher liver steatosis count than the normal diet group. Statistical analysis showed that there was a positive association between energy, fat, and fructose intake and liver steatosis cell count, while protein intake was negatively associated with liver steatosis. This finding is in line with previous studies that indicated that consumption of fructose and fat produced larger droplets of liver lipids and promoted increased liver fat. ${ }^{20,22}$ Furthermore, protein intake plays a role in improving liver steatosis, which is essential in the regeneration of hepatocytes and provides important amino acids that prevent liver fat deposition. This is demonstrated by studies using mung bean protein to reduce hepatic triglyceride concentration in 4 weeks. ${ }^{24,25}$ However, another study showed different finding in which higher total liver fat elevation occurred in rats given high-unsaturated fat diet than rats given high-saturated fat diet, which also means that high carbohydrate intake is also needed to affect metabolic control. ${ }^{19}$

This imbalance in macronutrient absorption can affect nutrient synthesis, degradation, and liver secretion in the liver that can induce liver fat accumulation, which results in liver steatosis and inflammation that lead to cardiovascular disease. The addition of fructose to drinking water may lead to the formation of triglyceride and VLDL via de novo lipogenesis in the liver by causing liver steatosis. Another mechanism by which fructose causes fatty liver disease is mediated by GLUT2, which transports fructose in the hepatocytes, which then undergoes phosphorylation into fructose1-phosphate by fructokinase and is split into glyceraldehyde and dihydroxyacetone phosphate by hepatic aldose. Both compounds can be converted into acetyl-CoA and enter the respiratory system to produce ATP or move as glycerol to produce triglyceride. Therefore, long-term fructose consumption may lead to metabolic impairment due to an increase in TG and accumulation of hepatic fat. 5,26,27 This study also reported that aortic wall thickness and foam cell count were significantly higher in HFFD than normal diet group. Protein intake is negatively associated with the 2 parameters, and the higher the energy, fat, and fructose intake, the higher the foam cell count and aortic wall thickness. This finding is in line with a previous study conducted in rats, which were given a high-fat diet for 6 months. At the end of the intervention, examination showed that the thickness of aorta rose in high-fat diet and that this increase was followed by endothelial damage in the form of alteration of endothelial lining and edema formation. ${ }^{28}$ Other studies also showed that high-fructose or high-fat diet given for 8 weeks resulted in the thickening of abdominal aortic in 32 Sprague Dawley rats and increased aorta lesion by $40 \%$ higher than the control group of 29 WHHL/Watanabe Heritable Hyperlipidemic rabbits. ${ }^{11,29}$ HFFD can also lead to the alteration of lipid serum, which is significantly associated with aorta thickening. This mechanism is stimulated by the rising of macrophage and smooth muscle cell resulting in aorta lesion and pathologic intimal thickening that lead into atherosclerosis plaque. ${ }^{1,11,29}$ Foam cell formation is mainly influenced by advanced glycation end products (AGE), which are formed endogenously through food due to the high intake of sugars, including fructose. Furthermore, AGE is also one of the factors responsible for diabetic nephropathy, which is associated with lipid accumulation. Carboxymethyl lysine as a member of AGEs increases lipid uptake and synthesis, and promote foam cell formation through increasing transcription and protein glycosylation of cleavage-activating protein in human mesangial cells. ${ }^{30}$ High-fat with fructose addition might have stronger effect on foam cell formation than high-fat alone. This is related with high expression of CCR7 (M1-phenotype) in advanced lesions located in the fibrous cap-like sites and M2-macrophages expressed abundantly in the foam-cell cores. ${ }^{31}$

Instead of using plant-based proteins such as soybeans, casein was used as a source of protein in this study because of its hypercholesterol-lowering effects as well as its effect on the formation of atherosclerotic lesions. In addition, it was reported that casein intake had a negative association with formation of atherosclerotic lesion $(r=-0.503)$. However, another study, which compared soybean and casein as protein source showed that casein had a higher probability of promoting atherosclerotic lesion formation. Therefore, the association between total casein intake and the occurrence of atherosclerosis is inconclusive. ${ }^{32,33}$

The histological examination of the heart showed higher values for inflammatory mononuclear and hypertrophic heart cells in the HFFD group, although there was no correlation between the intake of fat and fructose and the histological parameters of the heart. However, HFFD group clearly exhibited fibrotic presence and hypertrophic cell condition, which was also showed in some previous studies. ${ }^{14,34}$ This histology alteration is a manifestation of chronic inflammation, which starts from the inside of myocardium as the adaptive response, then causes activation of free radicals and chronic inflammation including cell hypertrophy. The chronic inflammation increases the production of TNF- $\alpha$, which induces the release of IL- 6 that leads to cell hypertrophy and also infiltration of the mononuclear cell. The formation of fibrosis also occurs in this state, starting from the increase of collagen from cardiac fibroblast as a result of chronic inflammation. Myocardial hypertrophy as a result of a high-fructose diet is caused by increased mitochondrial oxidative stress. This oxidative stress is stimulated by the decrease of cystic fibrosis transmembrane conductance regulator (CFTR) as the effect of a high-fructose diet. ${ }^{35,36}$

This study showed that protein intake was negatively associated with aorta thickness and cardiac histology parameter. However, the literature review shows mixed results, and another study observed that higher vegetable protein intake was linked to lower risk of coronary heart disease. While another study showed that animal-protein intake, with respect to its saturated fatty acid and cholesterol content, can increase mortality risk of cardiovascular diseases. This study, however, did not assess specifically the effect of different protein intake, and the difference results from variation in the composition of normal diet and HFFD feeding and feeding ingredients. The high-fat group can develop cardiac muscle injury caused by high oxidative stress, which also leads to lipotoxicity 
and abnormality in left ventricle contraction. Fibrosis occurs due to the increased infiltration of inflammatory cells to myocardium and upregulation of pro-inflammatory cytokines (IL-1 $\beta$ and TNF- $\alpha$ ) and adhesion molecules such (ICAM1 and VCAM). ${ }^{14,37,38}$

Global sugar consumption, including fructose, has been increasing over the past few decades along with the rise in Gross National Product (GNP) and urbanization. In addition, urbanization itself is highly correlated with easy access to processed food, which has high sugar content, as well as access to mass media, mass transportation, and modern market. ${ }^{39}$ In Indonesia, many surveys showed that the average sugar consumption per capital was $46 \mathrm{~g} /$ day. This is much higher than the national recommendation of $<25 \mathrm{~g}$ /day but is slightly lower than WHO recommendation of 50 $\mathrm{g}$ /day. Most sugars consumed were crystalized sugar, brown sugar, sweetened condensed milk, syrup, sweetened drink, and traditional snack. ${ }^{40}$ In general, fructose consumption poses higher risk of obesity than sucrose as it is more easily absorbed in the intestine. In addition, as its main metabolism occurs in the liver, fructose can be easily changed into to fructose- 1 phosphate, which is the precursor for triglyceride (TG) formation. Moreover, fructose can also be broken down into carbon molecules before reaching phosphofructokinase form. This molecules can transform into glycerol and fatty acid that lead to adiposity. ${ }^{9}$

Consuming fructose poses a greater risk of developing metabolic syndrome than consuming other sugars because fructose has a different intermediate metabolism in which fructose can be more easily removed from the plasma and then gets to the liver without insulin being metabolized. Although the metabolism of glucose via hexokinase and glycolysis is regulated by the energy state and the level of insulin in the cell, the metabolism of fructose does not require such regulations. It is broken down easily into glycolytic phase and then go to lipogenesis one. Fructose and sucrose content in the drink may have a higher effect on lipid serum than glucose. ${ }^{12}$ Therefore, it is important to limit fructose intake in relation to metabolic syndrome risk, and its restriction is known to decrease blood pressure, which may improve heart rate and cardiac output. Fructose restriction can also repair insulin sensitivity and glucose tolerance, although it is not directly related to body weight change. ${ }^{41}$

\section{Conclusions}

This study showed that HFFD had no difference in body weight gain and body fat index. Meanwhile, there was higher liver steatosis cell count, greater aortic wall thickness, higher foam cell count, and the presence of fibrotic in the cardiac cell. This research showed that high fructose consumption may promote early signs of cardiac disease characterized by atherosclerosis signed by cardiac histopathology deterioration and increase of foam cell and aorta thickness. Furthermore, HFFD consumption may stimulate liver steatosis without the occurrence of obesity as indicated by anthropometry measurement. This study encourages further exploration of the HFFD effect in relation to other metabolic changes such as plasma lipid profile or the composition of gut microbiota.
Correspondence: Dian Handayani, Department of Nutrition, Faculty of Medicine, Universitas Brawijaya, Malang, Indonesia, Jl. Veteran Malang, 65145, East Java, Indonesia.

Tel. +62341569117, Fax: +62341564755.

E-mail: handayani_dian@ub.ac.id

Key words: Atherosclerosis; body fat index; dietary fructose; liver steatosis; obesity

Contributions: DH, IK designed and coordinated the study; AMI, RMW, EF, ADK, SN, DN, ES, carried out the experiments and all biological assays; all authors actively prepared and conducted the data analysis and wrote the manuscript; $\mathrm{DH}$, assisted in data interpretation and contributed to the final manuscript.

Conflict of interests: All authors have declared no conflict of interest.

Acknowledgement: The author expresses profound gratitude to the Faculty of Medicine, Universitas Brawijaya, Malang, Indonesia for supporting this study.

Funding: This study was financially supported by Faculty of Medicine, Universitas Brawijaya (Decree: 28/SK/UN10.7/PN/ BPPM/2017).

Ethical approval: All procedures were approved by the research ethics committee of Faculty of Medicine, Universitas Brawijaya, Indonesia (Ethic Approval Number: 68/EC/KEPK/10/2017).

Conference presentation: Part of this study was presented at the $1^{\text {st }}$ International Nursing and Health Sciences Symposium, November $13^{\text {th }}$ to $15^{\text {th }} 2020$, Brawijaya University, Malang, Indonesia.

Received for publication: 14 January 2021.

Accepted for publication: 19 March 2021.

o Copyright: the Author(s), 2021

Licensee PAGEPress, Italy

Journal of Public Health Research 2021;10:2181

doi:10.4081/jphr.2021.2181

This work is licensed under a Creative Commons Attribution NonCommercial 4.0 License (CC BY-NC 4.0).

\section{References}

1. Suzuki K, Jayasena CN, Bloom SR. Obesity and appetite control. Exp Diabetes Res 2012;2012:1-9.

2. Sladoje DP, Kisić B, Mirić D. The monitoring of protein markers of inflammation and serum lipid concentration in obese subjects with metabolic syndrome. J Med Biochem 2017;36:366-74.

3. Csige I, Ujvárosy D, Szabó Z, et al. The impact of obesity on the cardiovascular system. J Diabetes Res 2018;2018:1-12.

4. Ahima RS. Metabolic syndrome: a comprehensive textbook. Cham: Springer; 2016.

5. Kucera O, Cervinkova Z. Experimental models of non-alcoholic fatty liver disease in rats. World $\mathrm{J}$ Gastroenterol 2014;20:8364.

6. Ministry of Health, Republic of Indonesia. Basic Health Research 2018. Jakarta: Ministry of Health Republic of Indonesia; 2018.

7. WHO. Obesity and overweight. Geneva: World Health Organization; 2020 [cited 2020 Sep 2]. Available from: https://www.who.int/en/news-room/fact-sheets/detail/obesity- 
and-overweight

8. WHO. Time to deliver: report of the WHO Independent Highlevel Commission on Noncommunicable Diseases. Geneva: WHO; 2018. Available from: https://apps.who.int/iris/handle/10665/272710

9. Bocarsly ME, Powell ES, Avena NM, et al. High-fructose corn syrup causes characteristics of obesity in rats: increased body weight, body fat and triglyceride levels. Pharmacol Biochem Behav 2010;97:101-6.

10. Sakakura K, Nakano M, Otsuka F, et al. Pathophysiology of atherosclerosis plaque progression. Hear Lung Circ 2013;22:399-411.

11. Ning B, Wang X, Yu Y, et al. High-fructose and high-fat dietinduced insulin resistance enhances atherosclerosis in Watanabe heritable hyperlipidemic rabbits. Nutr Metab (Lond) 2015;12:30.

12. Aeberli I, Hochuli M, Gerber PA, et al. Moderate amounts of fructose consumption impair insulin sensitivity in healthy young men: a randomized controlled trial. Diabetes Care 2013;36:150-6.

13. Ding S, Zhang C, Zhang L, et al. Fructose-fed induced metabolic syndrome model in cynomolgus monkeys. J Vet Sci Technol 2017;8:1-10.

14. Sahraoui A, Dewachter C, De Medina G, et al. Myocardial structural and biological anomalies induced by high fat diet in Psammomys obesus gerbils. PLoS One 2016;11:e0148117.

15. Supranto J. Teknik sampling untuk survei dan eksperimen. Jakarta: Rineka Cipta; 2000.

16. Reeves PG. Components of the AIN-93 diets as improvements in the AIN-76A diet. J Nutr 1997;127:838S-41.

17. Lee JS, Jun DW, Kim EK, et al. Histologic and metabolic derangement in high-fat, high-fructose, and combination diet animal models. Sci World J 2015;2015:1-9.

18. Handayani D, Chen J, Meyer BJ, et al. Dietary Shiitake mushroom (Lentinus edodes) prevents fat deposition and lowers triglyceride in rats fed a high-fat diet. J Obes 2011;2011:1-8.

19. Ble-Castillo JL, Aparicio-Trapala MA, Juárez-Rojop IE, et al. Differential effects of high-carbohydrate and high-fat diet composition on metabolic control and insulin resistance in normal rats. Int J Environ Res Public Health 2012;9:1663-76.

20. Pang J, Xi C, Huang X, Cui J, et al. Effects of excess energy intake on glucose and lipid metabolism in C57BL/6 mice. PLoS One 2016;11:e0146675.

21. Yang Y, Smith Jr DL, Keating KD, et al. Variations in body weight, food intake and body composition after long-term high-fat diet feeding in C57BL/6J mice. Obesity (Silver Spring) 2014;22:2147-55.

22. Burchfield JG, Kebede MA, Meoli CC, et al. High dietary fat and sucrose result in an extensive and time-dependent deterioration in health of multiple physiological systems in mice. $\mathrm{J}$ Biol Chem 2018;293:5731-5.

23. Wang CY, Liao JK. A mouse model of diet-induced obesity and insulin resistance. Methods Mol Biol 2012;821:421-33.

24. Watanabe H, Inaba Y, Kimura K, et al. Dietary mung bean protein reduces hepatic steatosis, fibrosis, and inflammation in male mice with diet-induced, nonalcoholic fatty liver disease. J Nutr 2017;147:52-60.

25. Ullah R, Rauf N, Nabi G, et al. Role of nutrition in the pathogenesis and prevention of non-alcoholic fatty liver disease: recent updates. Int J Biol Sci 2019;15:265.

26. Johnson RJ, Perez-Pozo SE, Sautin YY, et al. Hypothesis: could excessive fructose intake and uric acid cause type 2 diabetes? Endocr Rev 2009;30:96-116.

27. Johnson AMF, Olefsky JM. The origins and drivers of insulin resistance. Cell 2013;152:673-84.

28. Dziuba O. Blood coagulation and aortic wall integrity in rats with obesity-induced insulin resistance. Ukr Biochem J 2018;90:14-23.

29. Yoo S, Ahn H, Park YK. High dietary fructose intake on cardiovascular disease related parameters in growing rats. Nutrients 2016;9:11.

30. Yuan Y, Zhao L, Chen Y, et al. Advanced glycation end products (AGEs) increase human mesangial foam cell formation by increasing Golgi SCAP glycosylation in vitro. Am J Physiol Physiol 2011;301:F236-43.

31. Swier VJ, Tang L, Radwan MM, et al. The role of high cholesterol-high fructose diet on coronary arteriosclerosis. Histol Histopathol 2015;31:167-76.

32. Sakono M, Fukuyama T, Ni W-H, et al. Comparison between dietary soybean protein and casein of the inhibiting effect on atherogenesis in the thoracic aorta of hypercholesterolemic (ExHC) rats treated with experimental hypervitamin D. Biosci Biotechnol Biochem 1997;61:514-9.

33. Darioli R. Dietary Protein and Atherosclerosis. Int J Vitam Nutr Res 2011;81:153.

34. Panchal SK, Poudyal H, Iyer A, et al. High-carbohydrate highfat diet-induced metabolic syndrome and cardiovascular remodeling in rats. J Cardiovasc Pharmacol 2011;57:51-64.

35. Stansfield WE, Ranek M, Pendse A, Set al. The pathophysiology of cardiac hypertrophy and heart failure. In: Cellular and molecular pathobiology of cardiovascular disease. San Diego: Elsevier; 2014. p. 51-78.

36. Zhang Y-B, Meng Y-H, Chang S, et al. High fructose causes cardiac hypertrophy via mitochondrial signaling pathway. Am J Transl Res 2016;8:4869-80.

37. Ostrander DB, Sparagna GC, Amoscato AA, et al. Decreased cardiolipin synthesis corresponds with cytochromec release in palmitate-induced cardiomyocyte apoptosis. J Biol Chem 2001;276:38061-7.

38. Unger RH, Orci L. Lipoapoptosis: its mechanism and its diseases. Biochim Biophys Acta 2002;1585:202-12.

39. Popkin BM, Nielsen SJ. The sweetening of the world's diet. Obes Res 2003;11:1325-32.

40. Imanningsih N, Jahari AB, Permaesih ID, et al. Consumption and sources of added sugar in Indonesia: a review. Asia Pac J Clin Nutr 2018;27:47.

41. Lustig RH, Mulligan K, Noworolski SM, Tet al. Isocaloric fructose restriction and metabolic improvement in children with obesity and metabolic syndrome. Obesity 2016;24:45360 . 